\title{
Status and performance of the ATLAS MUON spectrometer
}

\author{
Sotirios Vlachos ${ }^{\mathrm{a}}$, on behalf of the ATLAS collaboration \\ ${ }^{a}$ National Technical University of Athens, 9 Iroon Polytechniou, GR-15780 Athens, Greece
}

\begin{abstract}
The ATLAS experiment at the Large Hadron Collider at CERN is currently waiting to record first data from protonproton collisions in 2009. During the last two years the MUON spectrometer was commissioned with cosmic rays while being assembled in the underground experimental cavern. In summer 2008, during the start-up phase of the LHC, the first events from single beams were recorded with the fully functional ATLAS detector. Subsequently the whole ATLAS detector was operated and commissioned with cosmic particles. The acquired data samples (of more than 100 million events, both with and without magnetic field) allowed a detailed study of the detector performance. In addition they were used to fine tune the spectrometer for an optimal operation with the first proton-proton collisions. Here the observed performance of the ATLAS MUON detection system is presented. Both the precision and the trigger chambers are studied with cosmic rays, as well as the level-1 muon trigger. First results from combined studies with other subdetectors are also presented.
\end{abstract}

Key words: Monitored drift tubes, Resistive plate chambers, Thin gap chambers, Cathode strip chambers

\section{Introduction}

The Large Hadron Collider (LHC) at CERN is designed to collide protons at a centre of mass energy of $14 \mathrm{TeV}$ at high luminosity. This will provide a powerfull probe for physics at the $\mathrm{TeV}$ scale for the first time.

ATLAS is one of the general purpose detectors built to study all aspects of proton-proton collisions. The expected physics coverage of the ATLAS detector is described in detail in [1]. Its muon spectrometer (MUON) is designed to achieve a momentum resolution of better than $10 \%$ for muon transverse momenta of up to $1 \mathrm{TeV}$. The spectrometer consists of a system of three superconducting air-core toroid magnets and is instrumented with three layers of Monitored Drift Tube chambers (Cathode Strip Chambers in the extreme forward region) acting as precision detectors. The requirement for maximal pseudorapidity coverage lead to a design of a triple system with one central barrel part plus two endcaps. Resistive Plate Chambers in the barrel and Thin Gap Chambers in the endcap regions provide a dedicated fast muon trigger system (as well as a measurement of second coordinate for muon tracks). The whole MUON spectrometer is equipped with a dedicated alignment system to allow a precise correlation between measurements of various detector parts. In addition a set of magnetic field sensors allow a realistic B-field reconstruction, necessary to achieve the design momentum resolution. A detailed presentation of the ATLAS detector can be found in [2].

Email address: S.Vlachos@cern.ch (Sotirios Vlachos)
LHC is expected to provide proton-proton collisions in 2009. However until then all ATLAS subsystems are operated and cosmic ray data are collected. In particular the MUON spectrometer is collecting cosmic muons for commissioning purposes as well as for performance studies. These muons offer a unique opportunity to evaluate the detector (in terms of efficiency, coverage, resolution etc.) before any proton-proton collisions are available. Several hundred millions of cosmic tracks have been recorded and analysed by the various subsystems. In this way the first collision data will be directly exploitable for physics studies.

In what follows the performance of the various MUON subsystems with cosmic muons is presented.

\section{Monitored Drift Tubes}

The MUON spectrometer is based on Monitored Drift Tubes (MDT) for precisely measuring particle trajectories. Since the end of $200899.8 \%$ of the chambers are operational with less than $0.1 \%$ dead channels. Less than $0.2 \%$ of the channels have more than $5 \%$ occupancy, hence the noise level in the detector is very limited.

The average MDT efficiency can be determined using cosmic muon tracks with track segments in two or more station layers (Inner/Middle/Outer). By selecting one layer, for example the Inner layer, and requiring a track with segments in the Middle and Outer layers which crosses the Inner layer, the number of missed segments in the Inner layer and the corresponding efficiency is determined. Note that the segment (here in the Inner Layer) is not required to lie on the track. The average MDT 
track segment efficiency for the barrel region is $0.97 \pm 0.01$. Figure 1 shows the residuals of fitting cosmic muon track segments using MDT hits. The distribution is fitted with two gaussians. The narrow one has a width of $105 \mu \mathrm{m}$. This is a very good estimation of the MDT measurement resolution.

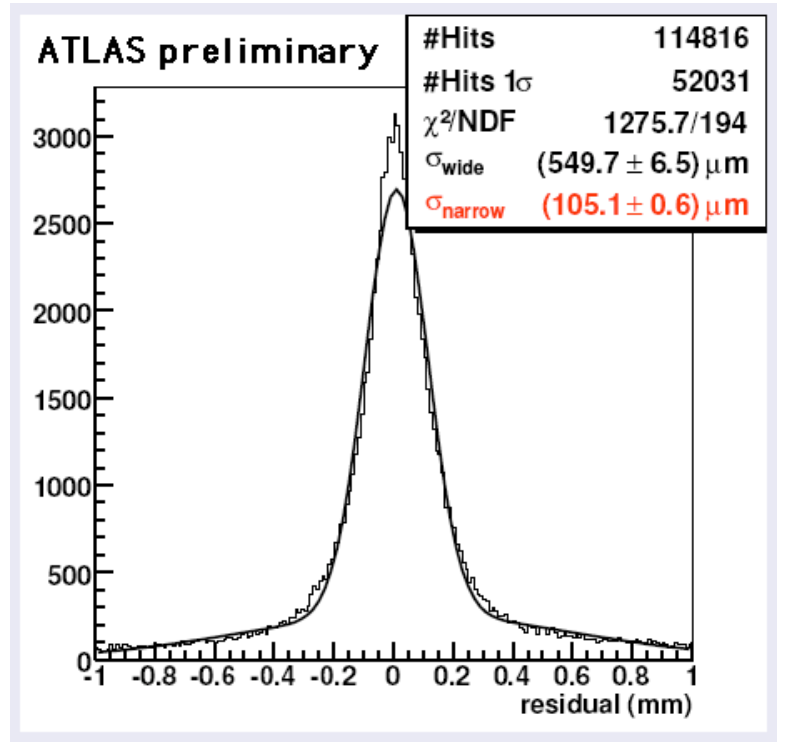

Figure 1: Residuals of MDT measurements of cosmic muon reconstructed tracks.

Using cosmic data acquired with the ATLAS magnets turned off, the performance of the MUON alignment system has also been studied. Reconstructed tracks in the Inner and Outer plane of the MUON spectrometer are extrapolated to the Middle plane and compared with track segments identified there. Without any alignment corrections the distance between measured and extrapolated segment (sagitta) is of the order of $2 \mathrm{~mm}$. Using the optical alignment system to correct the position of the MDT chambers the above sagitta is reduced to less than 150 $\mu \mathrm{m}$ for all MDT chambers. More details on the alignment system can be found in [3].

\section{Resistive Plate Chambers}

In the barrel region Resistive Plate Chambers (RPC) provide trigger and second coordinate information for through going particles. The RPC's time resolution allows the identification of the corresponding bunch crossing for each detected particle. In the end of 2008 95.5\% of the chambers were operational with less than $2 \%$ dead strips. Noise in the detector is limited to very low levels with less than $1 \%$ noisy channels at nominal operating conditions. Figure 2 shows a map of incoming cosmic muons. They are reconstructed using only RPC space point measurements. The tracks are projected at the surface $(\mathrm{y}=81 \mathrm{~m})$.

As far as trigger coverage is concerned, RPC were covering in 2008 approximately $70 \%$ of their design area. Pre- cise timing and fine tuning is ongoing to obtain full trigger coverage.

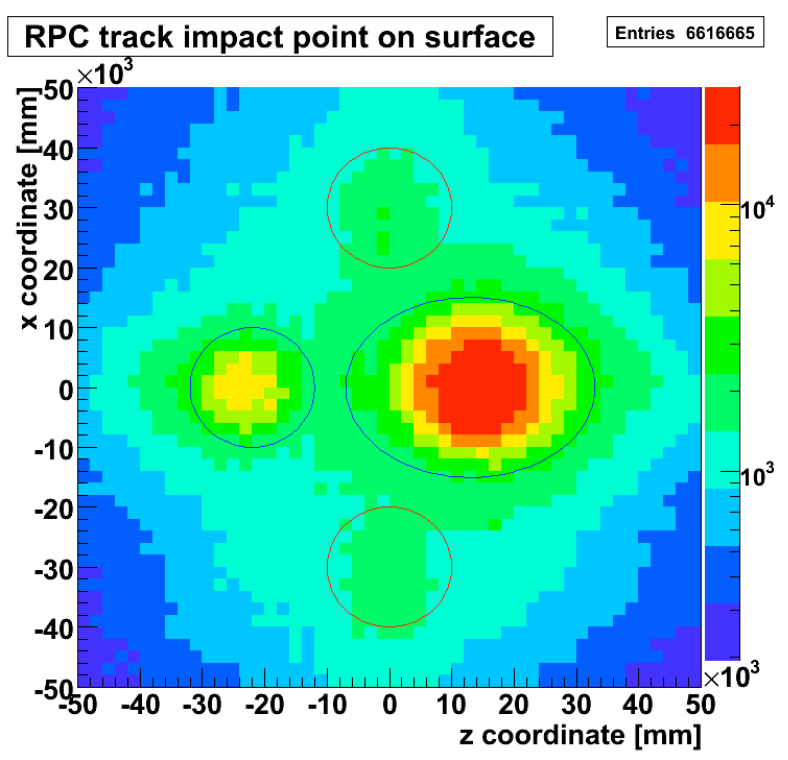

Figure 2: Cosmic muons reconstructed using the RPC 2-dimensional space point measurements. The tracks are extrapolated to the surface $(y=81 \mathrm{~m})$. The map shows clearly the two main shafts of the ATLAS experimental cavern (along $\mathrm{x}=0$ ) and to a lesser extent the two elevator shafts (along $\mathrm{z}=0$ )

\section{Thin Gap Chambers}

Thin Gap Chambers (TGC) are used for trigger and second coordinate measurement in the MUON endcaps. Currently $99.8 \%$ of the chambers are operational. They have less than $0.01 \%$ dead channels and a similar number of noisy channels (less than $0.02 \%$ of the TGC channels have more than $5 \%$ occupancy). Cabling maps, timing and operational parameters are all fine tuned to provide full trigger coverage for muons in the MUON endcaps. The efficiency of the detector is currently being evaluated with cosmic rays. Several independent estimations show efficiencies of above 0.85 for both wires and strips. These estimations include any loss of acceptance due to geometry effects.

Figure 3 shows the correlation between MDT tube hits in the middle station (out of the three MDT stations in each endcap) and TGC track interpolated to that plane. TGC tracks are reconstructed with wire hits and required at least 3 stations with hits.

\section{Cathode Strip Chambers}

In the very forward region of ATLAS Cathode Strip Chambers (CSC) instead of MDTs have been used. The choice was driven by the high radiation and particle flux in that region. All CSC chambers have been installed and commissioned in 2008. Cosmic data have shown less than 


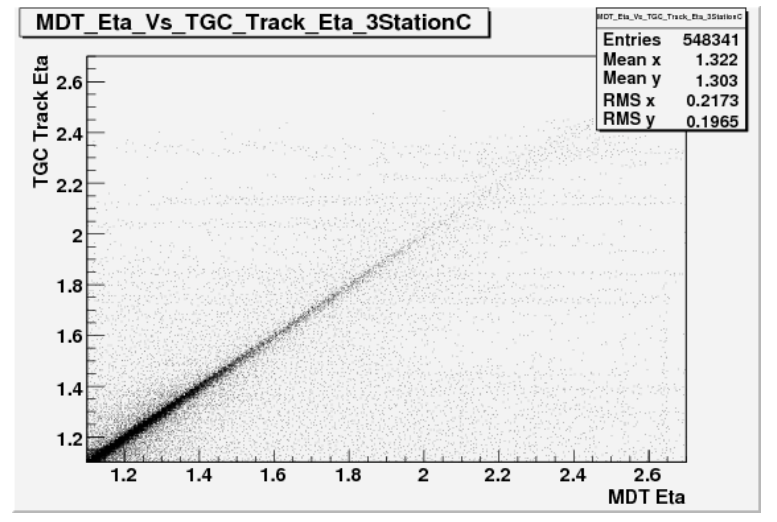

Figure 3: Correlation between MDT and TGC detectors for cosmic muons. The TGC reconstructed tracks are extrapolated to the middle MDT station and compared with the MDT precision measurements.

$0.1 \%$ dead channels. Precise calibration of the chambers with these data is ongoing. The 2008 cosmic data taking period has stressed the CSC data acquisition system to its limits. Several limitations have been identified which are currently being addressed. It is expected that the read out system will be fully operational and within specifications well before the first proton-proton collision data.

\section{Combined Performance}

The MUON spectrometer is only one of the many ATLAS subdetectors. For a thorough exploration of the ATLAS potential for physics measurements information from various subsystems has to be combined. As a first step in that direction the momentum of cosmic muons is measured with the MUON spectrometer and the ATLAS inner detector (ID) and the results are compared. Figure 4 shows the difference of these two measurements for tracks crossing both detectors. The peak of the distribution is not centred at 0 since between the two detectors lays the ATLAS calorimeter system. There cosmic muons are expected to lose on average $3 \mathrm{GeV}$. For completeness the expected (by Monte-Carlo simulation) momentum difference is also shown in the same figure. For this comparison events were required to have at least one ID track and at least one combined ID-MUON reconstructed track with 3 or more RPC hits. The comparison is between the momenta from the ID and the MUON standalone reconstruction.

\section{Summary}

During 2008 ATLAS and the MUON system studied the performance of the detector using cosmic rays. Several hundreds of millions of cosmic tracks were acquired and studied in detail. All the various MUON subsystems are fully operational and well performing. They are all ready for data taking with proton-proton collisions.

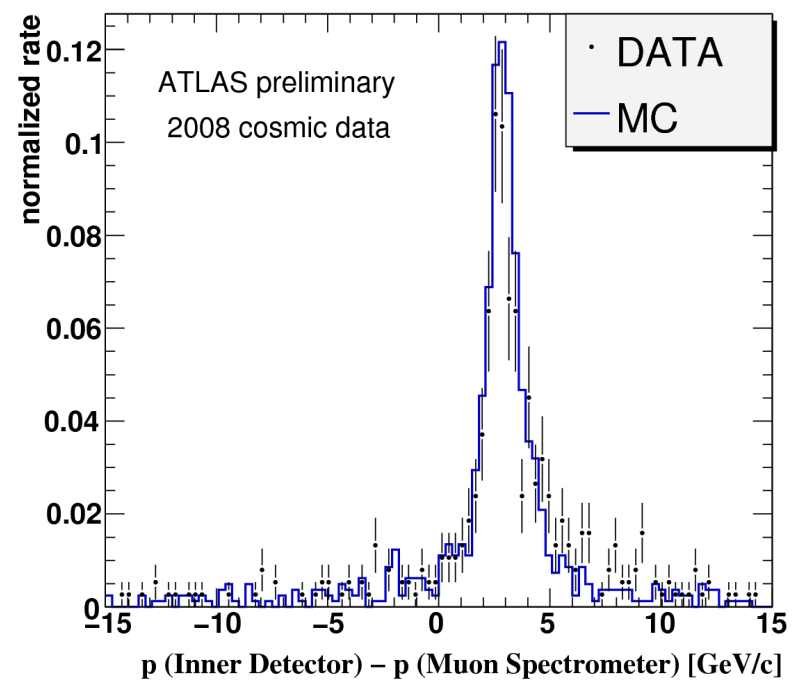

Figure 4: Difference between momentum measured by the MUON spectrometer and the ATLAS Inner detector, for cosmic muons. The peak is offset from zero due to the energy loss in the calorimeters between the two detectors. Both data and Monte-Carlo simulated events (MC) are shown for comparison.

\section{References}

[1] ATLAS Collaboration, 'Expected Performance of the ATLAS Experiment, Detector, Trigger and Physics', CERN-OPEN-2008020, Geneva, 2008.

[2] The ATLAS Collaboration, G. Aad et al., 'The ATLAS Experiment at the CERN Large Hadron Collider', JINST 3 (2008) S08003.

[3] C. Amelung for the ATLAS collaboration, 'First results from the ATLAS muon spectrometer optical alignment system', Presented in 'Technology and Instrumentation in Particle Physics', Tsukuba, Japan (March 2009). 